\title{
Intermittent sprint performance in the heat is not altered by augmenting thermal perception via L-menthol or capsaicin mouth rinses
}

\author{
O. R. Gibson ${ }^{1,2} \cdot$ J. G. Wrightson ${ }^{3} \cdot$ M. Hayes ${ }^{4}$
}

Received: 4 October 2018 / Accepted: 10 December 2018 / Published online: 22 December 2018

(c) The Author(s) 2018

\begin{abstract}
Purpose Cooling sensations elicited by mouth rinsing with L-menthol have been reported as ergogenic. Presently, responses to L-menthol mouth rinsing during intermittent sprint performance (ISP) in the heat are unknown and the impact of increased thermal perception on ISP via capsaicin has also not been quantified. This experiment aimed to identify whether eliciting cooling/warming sensations via L-menthol/capsaicin would alter ISP in the heat.

Method Fourteen participants (mass $=72 \pm 9 \mathrm{~kg}, \dot{V} \mathrm{O}_{2 \text { peak }}=3.30 \pm 0.90 \mathrm{~L} \mathrm{~min}^{-1}$ ), undertook four experimental trials, involving $40 \mathrm{~min}$ of ISP in hot conditions $\left(40.2 \pm 0.6^{\circ} \mathrm{C}, 42 \pm 2 \%\right.$ R.H.) with mouth rinsing $(25 \mathrm{~mL}, 6 \mathrm{~s})$ at the protocol onset, and every 10 min thereafter. Cooling ( $0.01 \%$ L-menthol; MEN), warming ( $0.2 \%$ capsaicin; CAP), placebo ( 0.3 sham-CHO; PLA), and control (water; CON) mouth rinses were utilized. Performance was quantified via power (PP) and work done (WD) during sprints. Heart rate (HR), core $\left(T_{\text {rec }}\right)$ and skin $\left(T_{\text {skin }}\right)$ temperature, perceived exertion (RPE), thermal sensation $\left(T_{\text {sens }}\right)$, and comfort $\left(T_{\text {com }}\right)$ were measured at $10 \mathrm{~min}$ intervals. Sweat rate (whole-body sweat rate) was calculated from $\Delta$ mass. Result PP reduced over time $(P<0.05)$; however, no change was observed between trials for PP or WD $(P>0.05)$. $T_{\text {com }}$ increased over time and was lower in MEN $(2.7 \pm 1.1 ; P<0.05)$ with no difference between CAP $(3.1 \pm 1.2)$, PLA $(3.2 \pm 1.3)$ and CON $(3.1 \pm 1.3)$. RPE, $T_{\text {sens }}$ HR, $T_{\text {rec }}$, and $T_{\text {skin }}$ increased over time $(P<0.05)$ with no between trial differences $(P>0.05)$. Conclusion Despite improved thermal comfort via L-menthol, ISP did not improve. Capsaicin did not alter thermal perception or ISP. The reduction in ISP over time in hot conditions is not influenced by thermal perception.
\end{abstract}

Keywords Cooling $\cdot$ Heat stress $\cdot$ Sprint performance $\cdot$ Temperature $\cdot$ Thermal comfort $\cdot$ Thermal sensation $\cdot$ Thermal perception

Communicated by George Havenith.

Electronic supplementary material The online version of this article (https://doi.org/10.1007/s00421-018-4055-0) contains supplementary material, which is available to authorized users.

O. R. Gibson

oliver.gibson@brunel.ac.uk

1 Centre for Human Performance, Exercise and Rehabilitation, College of Health and Life Sciences, Brunel University London, Uxbridge, UK

2 Division of Sport, Health and Exercise Sciences, Department of Life Sciences, College of Health and Life Sciences, Brunel University London, Uxbridge, UK

3 Faculty of Kinesiology, University of Calgary, Calgary, Canada

4 Environmental Extremes Laboratory, University of Brighton, Eastbourne, UK

\begin{tabular}{ll}
\multicolumn{2}{l}{ Abbreviations } \\
$T_{\text {rec }}$ & Core temperature \\
CISP & Cycling intermittent sprint protocol \\
$\mathrm{HR}$ & Heart rate \\
ISP & Intermittent sprint performance \\
$W_{\max }$ & Maximum aerobic power output \\
$\mathrm{MP}$ & Mean power \\
$\mathrm{NBM}$ & Nude-body mass \\
$\dot{V} \mathrm{O}_{2 \text { peak }}$ & Peak oxygen uptake \\
$\mathrm{PP}$ & Peak power \\
$\mathrm{RPE}$ & Rating of perceived exertion \\
$T_{\text {skin }}$ & Skin temperature \\
$T_{\text {com }}$ & Thermal comfort \\
$T_{\text {sens }}$ & Thermal sensation \\
TT & Time trial \\
TRP & Transient receptor potential channels \\
TRPM8 & TRP ion-channel melastatin 8 \\
TRPV1 & TRP ion-channel vanilloid 1
\end{tabular}


WBSR Whole-body sweat rate

WD Work done

\section{Introduction}

In comparison to equivalent exercise demands in temperate conditions, heat stress impairs endurance (Kenefick et al. 2007) and intermittent (Girard et al. 2015) exercise performance. In cycling (Racinais et al. 2015b) and running (Guy et al. 2014) modalities, continuous intensity/aerobic endurance-type exercise performance diminishes with increased environmental temperature along a continuum once the magnitude of heat stress increases above preferable ambient temperatures of $10-15^{\circ} \mathrm{C}$ (Galloway and Maughan 1997). Impaired performance of intermittent sprint activity replicating team sports, e.g., football/soccer, rugby league and union, field hockey, basketball and netball, during heat stress has been characterized (Hayes et al. 2014), albeit to a lesser extent than continuous exercise in spite of intermittent/repeated sprint exercise providing a greater heat strain (Maxwell et al. 1996). It has been reported that football/soccer players performing at the 2014 FIFA World Cup demonstrated a reduction in the number of sprints and total distance covered under high heat stress vs moderate or low heat stress (Nassis et al. 2015), an observation shared by others (Konefal et al. 2014; Watanabe et al. 2017). Conversely, within that same study, it was identified that technical parameters (rate of successful passes) were equal or enhanced in high heat stress vs moderate or low stress matches (Nassis et al. 2015), suggesting that 'pacing' of high-intensity work is occurring either in response to physiological or perceptual stimuli (Girard et al. 2017).

Detrimental performance under heat stress is closely related to the elevations in cardiovascular strain under heat stress (González-Alonso et al. 2008), which occur to attenuate elevations in core/deep body temperature via elevated skin blood flow and redistribution of cardiac output (González-Alonso 2012), ultimately to maximize evaporative cooling (Candas et al. 1979). Two experimental games of football played in hot $\left(\sim 43{ }^{\circ} \mathrm{C}\right)$ or temperate $\left(\sim 21{ }^{\circ} \mathrm{C}\right)$ conditions evidenced these responses with muscle and core temperature $\sim 1{ }^{\circ} \mathrm{C}$ higher in the hot games (Mohr et al. 2012; Nybo et al. 2013). These temperature responses were not accompanied by elevated cardiovascular strain. However, this is likely a response to aforementioned reductions in physical performance whereby distance covered $(-7 \%)$ and high-intensity running $(-26 \%)$ were lower in the hot conditions, presumably to moderate the magnitude of cardiovascular strain, i.e., the players were incurring similar physiological strain for reduced work. In a follow-up study utilising the same data (Nybo et al. 2013), no difference in maximal voluntary contraction, voluntary activation, and peak twitch torque, nor the magnitude of glycogen depletion occurred in the football matches played in the hot or temperate conditions, suggesting that these pathways are not directly contributing to reduced physical performance in the heat. Thermally driven pacing during intermittent team sport type activity has been evidenced in the laboratory, whereby relative to temperate conditions, hot-wet and hot-dry conditions elicit an earlier and greater reduction in peak power output during 40 min of high-intensity sprints (Hayes et al. 2014). The conscious/subconscious pacing of performance becomes apparent given equality of performance during the final sprint/end spurt across conditions inspite of earlier reductions in performance (Hayes et al. 2014). It has previously been identified that cooling at a physiological and perceptual level (Castle et al. 2006; Duffield et al. 2010) can enhance self-paced exercise performance under heat stress in laboratory conditions. This suggests, in these scenarios, that attenuated physiological or perceived temperature is ergogenic and, therefore, has the potential to improve intermittent sprint performance. Conversely, under conditions of equivalent core temperature, deception of temperature (Castle et al. 2012) and subsequent reductions in RPE leads to an improvement in performance, suggesting that perception of temperature is at least in part responsible for performance detriments rather than the physiological temperature alone (within the range of internal temperatures elicited by these experiments).

Eliciting alterations in thermal perception in the absence of any physiological differences in temperature has been termed 'non-thermal cooling' or 'non-thermal warming'. Non-thermal cooling can be administered by topical, facial applications of L-menthol [an activator of the transient receptor potential (TRP) ion-channel Melastatin 8 (TRPM8) (Montell and Caterina 2007)] to elicit alterations in thermal sensation to that of fan cooling, and capsaicin [an activator of the TRP ion-channel Vanilloid 1 (TRPV1) (Montell and Caterina 2007)] has been used to elicit non-thermal warming eliciting equivalent changes in thermal perception to that of a heater (Schlader et al. 2011). Non-thermal cooling sensation of L-menthol improved mean power output to the same extent as the actual cooling $(+21 \%$ during RPE-clamped exercise), whilst capsaicin and actual warming reduced power output relative to cooling trials (Schlader et al. 2011). These data inform that self-paced fixed-intensity exercise performance such as time trials or time to task failure/ exhaustion in the heat can be modulated by thermoregulatory behavior. Given the difficulties in applying non-thermal cooling via facial applications during fixed-intensity exercise performance, the ergogenic potential of non-thermal cooling via $L$-menthol in the form of a mouth rinse has received a significant attention. A recent review concluded that a mouth rinse or a beverage containing L-menthol during endurance exercise in the heat is beneficial for performance (Stevens 
and Best 2017). It was stated that ergogenic benefits for performance are contingent on altering thermal perception/perceived exertion (Mündel and Jones 2010; Stevens et al. 2016, 2017; Flood et al. 2017; Jeffries et al. 2018). This notion is supported by the observation that protocols which did not report altered perceptual responses following oral L-menthol do not demonstrate a performance enhancement (Sönmez et al. 2010; Riera et al. 2014, 2016). At present, only one experiment has investigated the use of L-menthol [combined with cool fluid $\left(0.2\right.$ or $\left.3.0^{\circ} \mathrm{C}\right)$ ] during interval-type activity (five repetitions of $4 \mathrm{~km}$ cycle and $1 \mathrm{~km}$ running TT performance), whereby no ergogenic improvement was attributable to L-menthol (Trong et al. 2015). To the authors' knowledge, no data exist investigating the use of L-menthol during intermittent sprint activity replicating that of team sports. This is surprising given that the natural breaks in play during team sport performance would facilitate mouth rinsing at the same time as habitual drinking (Garth and Burke 2013), something not as plausible in a continuous, fixed-intensity competitive endurance event such as track running/cycling.

The aim of this experiment was to determine whether eliciting a cooling sensation via oral L-menthol or a warming sensation via oral capsaicin would alter intermittent sprint performance in the heat in comparison to control and placebo oral solutions. It was hypothesized that L-menthol would reduce (enhance) thermal sensation and improve performance, whilst capsaicin would increase (diminish) thermal sensation and decrease performance.

\section{Methods}

\section{Participants}

Fourteen healthy, non-heat-acclimated, trained team sports players volunteered to participate in the study (Participant characteristics are presented in Table 1). Initially, 16 participants volunteered and commenced the experiment; however, two withdrew having completed two and three visits for reasons unrelated to the experiment. Confounding variables of caffeine and alcohol consumption $24 \mathrm{~h}$ prior to testing and prolonged thermal, e.g., exercise-heat acclimation protocols, repeated sauna or hot tub use, or hypoxic exposures, e.g., altitude training in the 6 weeks prior to testing, were all controlled for in line with the previous work involving exercise-heat stress and intermittent sprinting (Hayes et al. 2014; Gibson et al. 2014). Following institutional ethics approval (2732-MHR-Jul/2016-3430-2) and full description of experimental procedures, all participants completed medical questionnaires and provided written informed consent following the principles outlined by the declaration of Helsinki of 1975, as revised in 2013.
Table 1 Mean \pm SD participant characteristics $(n=14 ; 11$ males; 3 females)

\begin{tabular}{lc}
\hline Variable & Mean $\pm \mathrm{SD}$ \\
\hline Age (years) & $24 \pm 3$ \\
Height $(\mathrm{cm})$ & $175 \pm 12$ \\
Mass $(\mathrm{kg})$ & $71.6 \pm 8.8$ \\
Body surface area $\left(\mathrm{m}^{2}\right)$ & $1.86 \pm 0.17$ \\
Body mass index $\left(\mathrm{kg} \mathrm{m}^{2}\right)$ & $23.4 \pm 2.2$ \\
Body fat $(\%)$ & $11.6 \pm 3.1$ \\
Maximal oxygen uptake $\left(\mathrm{L} \mathrm{min}^{-1}\right)$ & $3.29 \pm 0.89$ \\
Maximal oxygen uptake $\left(\mathrm{mL} \mathrm{kg}^{-1} \mathrm{~min}^{-1}\right)$ & $46.2 \pm 12.9$ \\
Power at maximal oxygen uptake $\left(\mathrm{W} \mathrm{kg}^{-1}\right)$ & $3.8 \pm 1.3$ \\
Power eliciting $35 \% \dot{V} \mathrm{O}_{2 \text { peak }}\left(\mathrm{W} \mathrm{kg}^{-1}\right)$ & $1.3 \pm 0.5$ \\
\hline
\end{tabular}

\section{Experimental design}

The protocol consisted of five visits. The first visit was an incremental test to determine peak oxygen uptake $\left(\dot{V} \mathrm{O}_{2 \text { peak }}\right)$ and maximum aerobic power output ( $\left.W_{\max }\right)$. During the same visit, the subjects completed a familiarization trial of $20 \mathrm{~min}$ of a cycling intermittent sprint protocol [CISP; The full CISP is $40 \mathrm{~min}$ in duration, and involves $20 \times 2 \mathrm{~min}$ blocks of $10 \mathrm{~s}$ passive rest, a $6 \mathrm{~s}$ sprint against $7.5 \%$ of body mass, and $104 \mathrm{~s}$ of active recovery at a power eliciting $35 \%$ $\dot{V} \mathrm{O}_{2 \text { peak }}$ determined from regression of $\dot{V} \mathrm{O}_{2}$ against power from the incremental test (Castle et al. 2011)] in hot conditions $\left[40{ }^{\circ} \mathrm{C}, 50 \%\right.$ relative humidity $\left.(\mathrm{RH})\right]$. This served to minimize the negative effects of initial heat exposure and any subject learning effect associated with the protocol (Hayes et al. 2014). Then, a minimum of $48 \mathrm{~h}$ later, in a randomized and cross-over design, participants commenced the experimental trials (visits 2-5). Female participants performed experimental trials during the follicular phase of the menstrual cycle (Mee et al. 2015; Lei et al. 2016). Trial order was counterbalanced using a Latin square based on 16 participants. Each experimental visit to the laboratory was separated by a minimum of $48 \mathrm{~h}$ to allow for a full-recovery between trials, and to mitigate against physiological adaptation to the heat (Gill and Sleivert 2001). All exercise tests were carried out on a friction braked cycle ergometer (Monark 724, Vansbro, Sweden), operating in a pedal-rate independent mode.

Experimental trials involved participants performing a CISP in a heat chamber maintained at $\sim 40{ }^{\circ} \mathrm{C}, \sim 50 \% \mathrm{RH}$. In the experimental trials, subjects periodically swilled an L-menthol, capsaicin, water, or orange-flavored placebo solution at four time points. Each mouth rinse was $25 \mathrm{~mL}$ in volume and administered at rest and at 10 min intervals thereafter, i.e., after every 5 th sprint during the $10 \mathrm{~s}$ passive rest phase of the CISP protocol. The participants were instructed to swill/gargle the solution around the mouth for 
$5 \mathrm{~s}$ and then expectorate the solution into a bowl without swallowing. The menthol solution (MEN) was a $0.01 \%$ concentration of L-menthol crystal ground and dissolved in distilled water ( $\geq 99 \%$ food grade L-menthol, Sigma-Aldrich, UK) in line with the previous experiments (Mündel and Jones 2010; Stevens et al. 2016). The capsaicin solution (CAP) was administered at a concentration of $0.2 \%$ with the capsaicin containing red pepper sauce (Tabasco Habanero Sauce, McIlhenny Co., Avery Island, CA, USA) diluted in distilled water. These differences in concentrations were used based upon pilot data which identified them to elicit an equal magnitude of perceptual change in thermal sensation of the oral cavity +0.5 (MEN) or -0.5 (CAP) on the scale (Toner et al. 1986) at rest, in thermoneutral conditions. Orange-flavored fruit squash (Tesco Ltd, UK) solution mixed with distilled water to create a $0.5 \%(0.3 \mathrm{~g} / 100 \mathrm{~mL})$ placeboCHO solution (PLA) (Carter et al. 2004). Distilled water served as the control (CON). To minimize the impact of drink temperature on perceptual or physiological responses (Lee and Shirreffs 2007), all fluids were maintained to the temperature within the heat chamber $\left(\sim 40^{\circ} \mathrm{C}\right)$, this also served to ensure that the L-menthol crystals remained dissolved in water and ensured minimal pre/per-cooling effect given the close proximity of the rinse temperature to that of the oral cavity.

\section{Preliminary testing}

Prior to the initial assessment of $\dot{V} \mathrm{O}_{2 \text { peak }}$ and $W_{\text {max }}$ in the preliminary trial, standing height $(\mathrm{cm})$ was measured via a stadiometer and nude-body mass $(\mathrm{kg})$ was recorded following self-reported measurement in a private bathroom (SECA 875 scale, Birmingham, UK). These data were used to calculate body surface area (Du Bois and Du Bois 1916). Body density was also calculated using calipers (Harpenden, Burgess Hill, UK) and a four-site skinfold calculation (Durnin and Womersley 1974). This was later used to calculate body fat $[\%$, (Siri 1956)].

The incremental test was conducted in temperate lab conditions $\left(21{ }^{\circ} \mathrm{C}, 50 \% \mathrm{RH}\right)$. Starting intensity was set at $80 \mathrm{~W}$ with resistance subsequently applied to the flywheel to elicit a $24 \mathrm{~W} \mathrm{~min}{ }^{-1}$ increase at the constant cadence of $80 \mathrm{rpm}$. Expired metabolic gas was measured using online gas analysis (Oxycon Pro, Jaeger GmbH or Metalyser Sport, Cortex, Leipzig, Germany); the $\dot{V} \mathrm{O}_{2 \text { peak }}$ was considered as the highest $\dot{V} \mathrm{O}_{2}$ obtained in any $10 \mathrm{~s}$ period. Heart rate (HR;

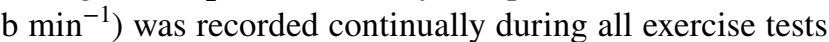
by short-range telemetry (Polar Electro Oyo, Temple, Finland). Saddle position was adjusted by the participant to their preferred cycling position and remained unchanged for all experimental trials. Experimental workloads (i.e., active recovery at $35 \%$ of $\dot{V} \mathrm{O}_{2 \text { peak }}$ ) were subsequently calculated using linear regression utilising power: $\dot{V} \mathrm{O}_{2}$ data collected following the incremental test.

After $25 \pm 5 \mathrm{~min}$ recovery, a $20 \mathrm{~min}$ stabilization period (seated rest), and ten stages (20 $\mathrm{min})$ of the CISP were completed in the heat chamber $\left(\sim 40{ }^{\circ} \mathrm{C}, \sim 50 \% \mathrm{RH}\right)$. Following stabilisation, a standard warm-up [5 min at $95 \mathrm{~W}(80 \mathrm{rpm})$ and two $30 \mathrm{~s}$ bouts at $120 \mathrm{~W}(100 \mathrm{rpm})$ with $30 \mathrm{~s}$ rest in between (Hayes et al. 2012, 2014)] were followed by the $10 \times 2$ min blocks of the CISP, i.e., ten instances of $10 \mathrm{~s}$ passive rest, a $6 \mathrm{~s}$ sprint against $7.5 \%$ of body mass, and $104 \mathrm{~s}$ of active recovery at a power eliciting $35 \% \dot{V} \mathrm{O}_{2 \text { peak }}$ (Castle et al. 2011).

\section{Experimental procedure}

Each experimental trial was conducted at the same time of day (within participants) to control for the effects of circadian variation in performance (Drust et al. 2005). To minimize differences in starting muscle glycogen concentrations between visits, subjects recorded their diet in the 24-h period before the second visit and were instructed to follow the same diet before each subsequent visit. Energy or macronutrient intake was not quantified in the experiment. Participants consumed $500 \mathrm{~mL}$ of water $2 \mathrm{~h}$ before all preliminary and experimental exercise sessions to ensure adequate hydration. Once participants were deemed euhydrated [i.e., urine osmolality (Alago Vitech Scientific, Pocket PAL-OSMO, UK) was $<700 \mathrm{mOsm} \mathrm{kg}{ }^{-1} \mathrm{H}_{2} \mathrm{O}$ (Sawka et al. 2007)], they were subsequently able to commence further preliminary and experimental procedures. Following confirmation of adequate hydration, in private, participants measured their nude-body mass (NBM) inserted a single-use disposable rectal thermistor (Henleys Medical, UK, Meter logger Model 401, Yellow Springs Instruments, Yellow Springs, Missouri, USA) $10 \mathrm{~cm}$ past the anal sphincter to facilitate the measurement of rectal temperature $\left(T_{\text {rec }}\right)$. Skin temperature $\left(T_{\mathrm{sk}}\right)$ was measured using a data logger (Squirrel Meter Logger, Grant Instruments, Cambridge, UK) and skin thermistors secured at four sites (pectoralis major muscle belly, lateral head of triceps brachii, rectus femoris muscle belly, and lateral head of the gastrocnemius) on the right-hand side of the body using $6 \mathrm{~cm} \times 7 \mathrm{~cm}$ transparent Tegaderm patches (3M, UK). Weighted mean skin temperature was determined using a four-site formula (Ramanathan 1964). Heart rate was recorded continually in the same manner as the preliminary test.

Participants then mounted a cycle ergometer located inside the environmental chamber where conditions were consistent across trials $\left(\sim 40{ }^{\circ} \mathrm{C}, \sim 50 \% \mathrm{RH}\right)$ and performed the warm-up followed by the full $40 \mathrm{~min}$ CISP. During the CISP, the Monark Anaerobic software (Monark Anaerobic Wingate Software, Version 1.0, Monark, Vansbro, 
Sweden) recorded peak power (PP), mean power (MP), and work done (WD) at a sampling frequency of $50 \mathrm{~Hz}$, during each sprint within of each 2 min stage of the CISP. Peak power was recorded as the highest recorded power output value for each sprint; MP was determined as the average power output from all values recorded during the $6 \mathrm{~s}$ sprint. WD was calculated cumulatively over the whole protocol. Physiological measurements were recorded at rest $(0 \mathrm{~min})$ and then $60 \mathrm{~s}$ into the active recovery phase after every 5 th sprint (every $\sim 10 \mathrm{~min}$ ) thereafter. Perceptual responses were also recorded at $10 \mathrm{~min}$ intervals with this frequency least likely to cause non-experimental artifacts (Corbett et al. 2009). Perceptual responses included whole-body thermal comfort $\left(T_{\text {com }}\right)$ and thermal sensation $\left(T_{\text {sen }}\right)$ determined on a 5- (from 1, comfortable, to 5, very uncomfortable) and 17- (from 0.0, unbearably cold, to 8.0 , unbearably hot) point scale, respectively (Toner et al. 1986), and the rating of perceived exertion (RPE) measured using a 15-point Borg scale (from 6, very very light, to 20, very very hard) (Borg 1982). Following completion of the protocol, the participants' towel dried and recorded NBM for the later calculation of whole-body sweat rate (WBSR, $\mathrm{L} \mathrm{h}^{-1}$ ).

\section{Data analysis}

Data are presented as mean $\pm \mathrm{SD}(n=14)$ unless, otherwise, indicated. All statistical analyses were carried out using the SPSS software (Version 25). All outcome variables were first checked for normality and sphericity. The Greenhouse-Geisser correction for the $F$ statistic and related degrees of freedom was used when data violated sphericity. The data from the CISP were "blocked" into an average of each 10 min series of sprints/recovery sprints. A two-way repeated-measures analysis of variance (ANOVA) was performed to determine differences in dependent variables associated with performance (PP, MP; $\mathrm{W} \mathrm{kg}^{-1}$ ) during each of the four 10 min blocks between the four mouth-rinse trials. Perceptual measures of RPE, $T_{\text {sen }}, T_{\text {com }}$, and physiological measures of $T_{\text {rec }}, T_{\text {skin }}$, and HR were compared between the four trials, and across five timepoints $(0,10,20$, 30, $40 \mathrm{~min}$ ). WBSR and WD were analyzed using a one-way repeated-measures ANOVA between the four mouth-rinse trials. Main and interaction effects were followed up with Bonferroni adjusted post hoc comparisons. Partial Eta squared $\left(\eta_{\mathrm{p}}^{2}\right)$ was used as an estimate of the effect size for main and interaction effects $[0.01=$ small, $0.06=$ medium, and $0.013=$ large (Cohen 1992)]; Cohen's $d_{\mathrm{av}}\left(d_{\mathrm{av}}\right)$ was used as an estimate of the effect size [0.2 $=$ small, $0.5=$ medium, and $0.8=$ large (Cohen 1992)] for post hoc comparisons. The threshold for rejecting the null hypothesis was set at $P<0.05$.

\section{Results}

\section{Effect of mouth rinse on sprint performance}

There was no main effect of mouth rinse, or interaction effect between mouth rinse and time, on peak power (Fig. 1), mean power or total work done (Fig. 2) [PP mouth rinse: $F_{(2,27)}<0.1, P=0.967, \eta_{\mathrm{p}}^{2}=0.003$, mouth rinse $\times$ time: $F_{(4,54)}=0.4, P=0.776, \eta_{\mathrm{p}}^{2}=0.034$, MP mouth rinse: $F_{(3,39)}<0.1, P=0.981, \eta_{\mathrm{p}}^{2}=0.005$, mouth rinse $\times$ time: $F_{(4,51)}=0.9, P=0.447, \eta_{\mathrm{p}}^{2}=0.067$, work done (kJ) mouth rinse: $\left.F_{(3,39)}=0.4, P=0.735, \eta_{\mathrm{p}}^{2}=0.032\right]$.

Peak power $\left(\mathrm{W} \mathrm{kg}^{-1}\right)$ and mean power $\left(\mathrm{W} \mathrm{kg}^{-1}\right)$ decreased during the first three blocks [main effect of time, PP: $F_{(1.3,16)}=10.6, P=0.003, \eta_{\mathrm{p}}^{2}=0.450, \mathrm{MP}: F_{(1,15)}=14.3$, $\left.P=0.001, \eta_{\mathrm{p}}^{2}=0.524\right]$. PP and MP were significantly lower during sprints $6-10$ compared to sprints 1-5 (PP: $P=0.027, d_{\mathrm{av}}=0.3$, MP: $\left.P=0.018, d_{\mathrm{av}}=0.3\right)$, and significantly lower during sprints 11-15 compared to sprints 6-10 (PP: $P=0.002, d_{\mathrm{av}}=0.3$, MP: $P<0.001, d_{\mathrm{av}}=0.3$ ). Sprint performance data are presented in Figs. 1 and 2 with data tables included as electronic supplementary material.

\section{Perceptual responses to mouth rinse}

There was a main effect of mouth rinse on $T_{\text {com }}\left[F_{(3,39)}=2.9\right.$, $\left.P=0.046, \eta_{\mathrm{p}}^{2}=0.183\right]$, where $T_{\text {com }}$ was lower after L-menthol $(2.7 \pm 1.1)$ than after carbohydrate mouth rinse $(3.2 \pm 1.3$, $\left.P=0.042, d_{\mathrm{av}}=0.4\right)$. There was no difference between any other mouth-rinse conditions, and no interaction effect between mouth rinse and time $\left[F_{(6,82)}=0.8, P=0.548\right.$, $\left.\eta_{\mathrm{p}}^{2}=0.061\right]$.

$T_{\text {com }}\left[F_{(2,31)}=121.0, P<0.0001, \eta_{\mathrm{p}}^{2}=0.903\right], T_{\text {sen }}$ $\left[F_{(2,20)}=216.1 \quad P<0.0001, \eta_{\mathrm{p}}^{2}=0.943\right]$ and $\mathrm{RPE}$ $\left[F_{(2,28)}=399.1, P<0.0001, \eta_{\mathrm{p}}^{2}=0.968\right]$ increased throughout each trial where all perceptual responses were higher than at the previous time point $\left(T_{\text {com }}\right.$; all $P<0.003, d_{\text {av }}>0.6, T_{\text {sen }}$; all $P<0.0001, d_{\mathrm{av}}>0.7$, RPE; all $\left.P<0.0001, d_{\mathrm{av}}>0.7\right)$. Perceptual responses to mouth rinse are displayed in Fig. 3 with data tables included as electronic supplementary material.

There was no main effect of mouth rinse and no interaction between mouth rinse and time on $T_{\text {sen }}$ or RPE [ $T_{\text {sen }}$ mouth rinse: $F_{(3,39)}=0.6, P=0.639, \eta_{\mathrm{p}}^{2}=0.042$, mouth rinse $\times$ time: $F_{(5,66)}=1.2, P=0.313, \eta_{\mathrm{p}}^{2}=0.085$, RPE mouth rinse: $F_{(2,26)}=0.6, P=0.543, \eta_{\mathrm{p}}^{2}=0.046$, mouth rinse $\times$ time: $\left.F_{(12,156)}=1.2, P=0.289, \eta_{\mathrm{p}}^{2}=0.084\right]$. 
Fig. 1 Mean $\pm 95 \%$ CI Peak power output during the sprint blocks across the menthol, capsaicin, carbohydrate, and water mouth-rinse conditions. Asterisk denotes difference from the previous sprint block with no difference between groups

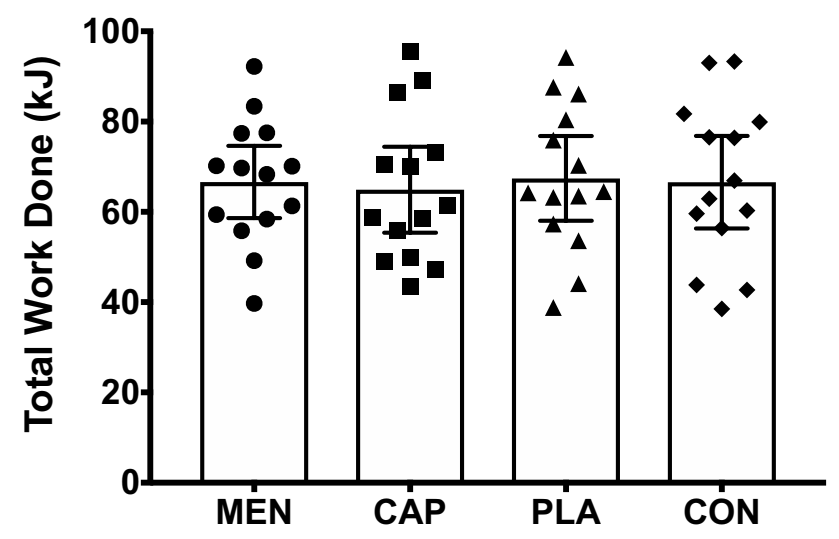

Fig. 2 Mean $\pm 95 \%$ CI total work done during sprints across the menthol, capsaicin, carbohydrate, and water mouth-rinse conditions

\section{Physiological responses to mouth rinse}

There was no main effect of mouth rinse, or interaction effect between mouth rinse and time, on $T_{\text {rec }}, T_{\text {skin, }}$ HR or WBSR [ $T_{\text {rec }}$ : mouth rinse: $F_{(3,39)}<0.1, P=0.967, \eta_{\mathrm{p}}^{2}=0.003$, mouth rinse $\times$ time: $F_{(3,44)}=1.1, P=0.355, \eta_{\mathrm{p}}^{2}=0.079, T_{\text {skin }}$ mouth rinse: $F_{(3,39)}=0.1, P=0943, \eta_{\mathrm{p}}^{2}=0.010$, mouth rinse $\times$ time: $F_{(3,44)}=0.9, P=0.465, \eta_{\mathrm{p}}^{2}=0.064$, HR mouth rinse: $F_{(3,39)}=0.4, P=0.769, \eta_{\mathrm{p}}^{2}=0.028$, mouth rinse $\times$ time: $F_{(4,56)}=0.5, P=0.769, \eta_{\mathrm{p}}^{2}=0.035$, WBSR mouth rinse: $\left.F_{(3,39)}=0.2, P=0.925, \eta_{\mathrm{p}}^{2}=0.012\right]$. Physiological responses are presented in Figs. 4 and 5 with data tables included as electronic supplementary material.
*

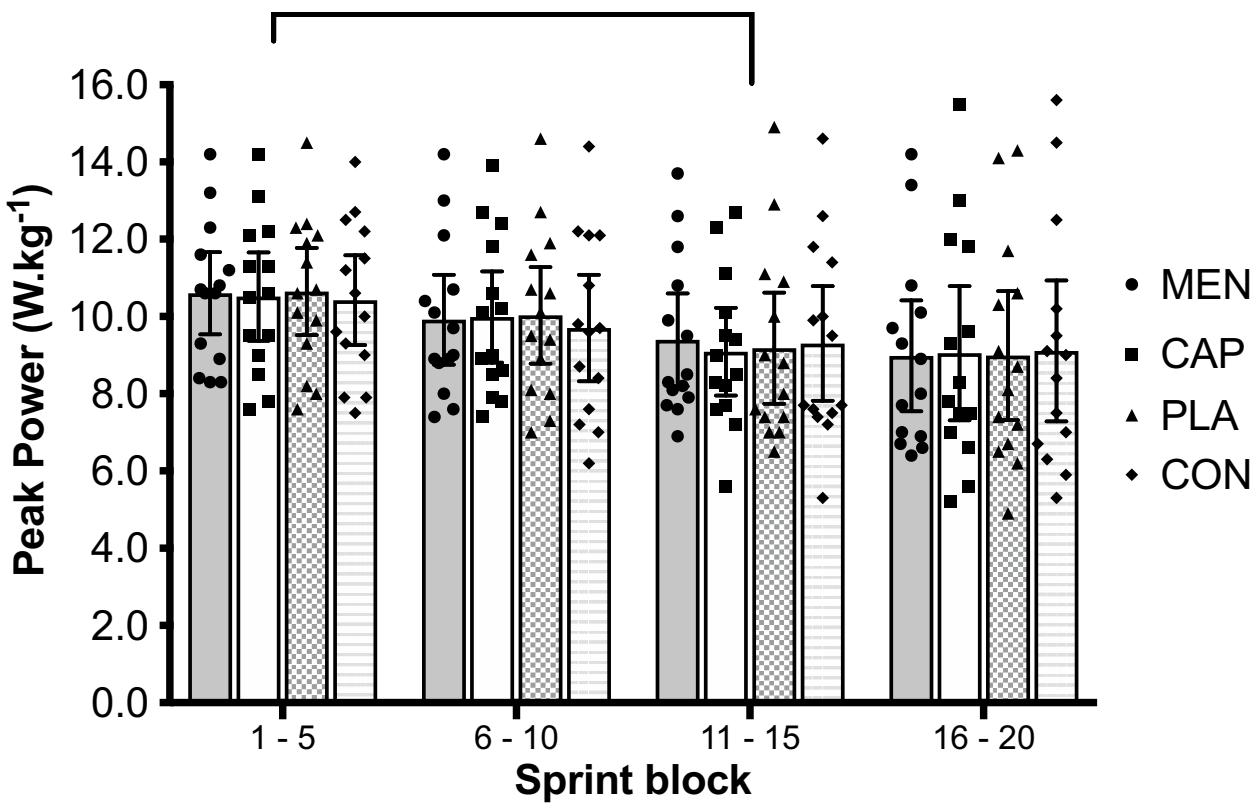

Physiological responses are presented in Figs. 4 and 5 with data tables included as electronic supplementary material. $T_{\text {rec }}\left({ }^{\circ} \mathrm{C}\right), T_{\text {skin, }}\left({ }^{\circ} \mathrm{C}\right)$, and HR $\left(\mathrm{b} \mathrm{min}{ }^{-1}\right)$ increased over time $T_{\text {rec }}: F_{(1,15)}=150.7, P<0.001, \eta_{\mathrm{p}}^{2}=0.921, T_{\text {skin }}$ : $F_{(2,21)}=78.4, P=0.001, \eta_{\mathrm{p}}^{2}=0.858, \mathrm{HR}:\left(F_{(2,26)}=294.6\right.$ $\left.P<0.001, \eta_{\mathrm{p}}^{2}=0.958\right]$.

$T_{\text {rec }}$ was higher at every time point than the previous time point (all $P<0.001$, all $d_{\mathrm{av}}>0.8$ ). The same trend occurred for $T_{\text {skin }}$ and HR (all $P<0.02, d_{\mathrm{av}}>0.4$ ), though the differences between $20 \mathrm{~min}$ and $30 \mathrm{~min}\left(T_{\text {skin }}\right.$ : $P=0.051, d_{\mathrm{av}}=0.3$, HR: $\left.P=0.316, d_{\mathrm{av}}=0.3\right)$ were not statistically significant.

\section{Discussion}

The aim of this experiment was to determine whether eliciting a cooling sensation via oral L-menthol or a warming sensation via oral capsaicin would alter intermittent sprint performance in the heat in comparison to control and placebo oral solutions. Identical physiological responses (Figs. 4, 5) between mouth-rinse conditions demonstrate equality of the physical capacity to perform intermittent sprints in the heat. Enhanced thermal comfort $[-0.5$ vs PLA $(P<0.05)$, and -0.4 vs CAP and CON] following L-menthol stimulation of the TRPM8 ion channel (Fig. 3) suggested that the participants were more 'perceptually tolerant' of the physiological heat stress, therefore, in line with the work of others, which had the potential to perform better given a reduction in perceived heat stress. This improved thermal perception via 

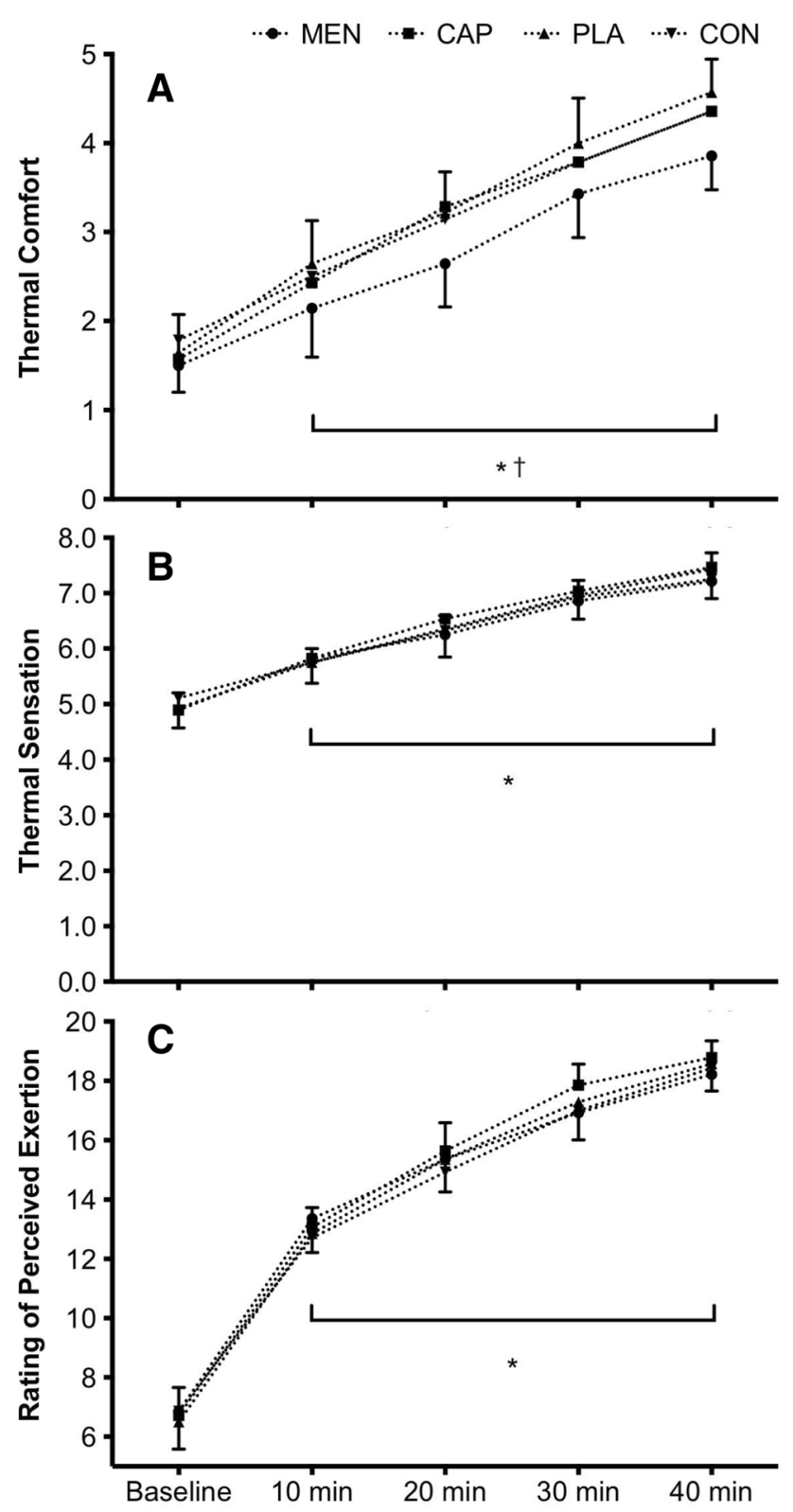

Fig. 3 Mean $\pm 95 \%$ CI thermal comfort (a), thermal sensation (b), and rating of perceived exertion (c) across the menthol, capsaicin, carbohydrate, and water mouth-rinse conditions, for all time points. Asterisk denotes difference from the previous sprint block. Dagger denotes significant difference in menthol

L-menthol did not, however, alter intermittent sprint performance (Figs. 1, 2). Opposing the experimental hypothesis, the capsaicin mouth rinse elicited no detrimental effect on performance, perception, or physiological responses with all data comparable to the placebo and control. Accordingly, the targeted TRPV1 channel was insufficiently modulated by our capsaicin rinse protocol.

Our data contrast the evidence of performance enhancement during fixed-intensity tasks following oral menthol interventions from cycling time to exhaustion/task failure

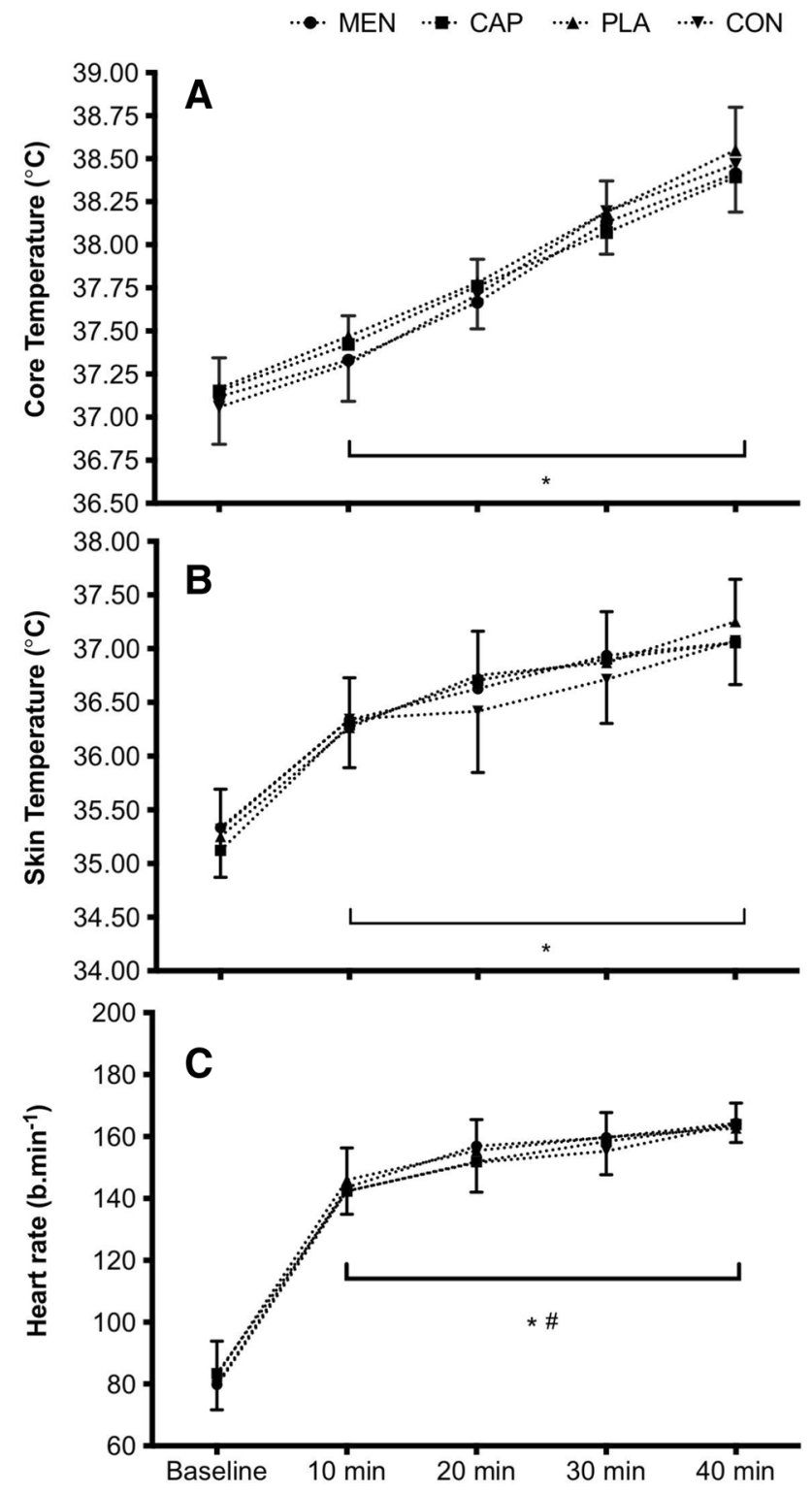

Fig. 4 Mean $\pm 95 \%$ CI core temperature (a), skin temperature (b), and heart rate (c) across the menthol, capsaicin, carbohydrate, and water mouth-rinse conditions, for all time points. Asterisk denotes difference from the previous sprint block with no difference between groups

experiments [+9\% (Mündel and Jones 2010), + 8\% (Flood et al. 2017) and $+6 \%$ (Jeffries et al. 2018)] and pre-loaded running time trials of $3 \mathrm{~km}$ and $5 \mathrm{~km}$ [TT; $-3 \%$ (Stevens et al. 2016) and -3.5\% (Stevens et al. 2017)]. The common theme amongst the studies demonstrating a benefit of Menthol are reports of improved thermal perception (Stevens et al. 2016, 2017; Flood et al. 2017; Jeffries et al. 2018) or reduced perceived exertion (Mündel and Jones 2010). Our data add to the equivocal findings relating to the use of oral Menthol interventions which have reported no ergogenic effects during performance trials, e.g., $400 \mathrm{~m}$ running TT 


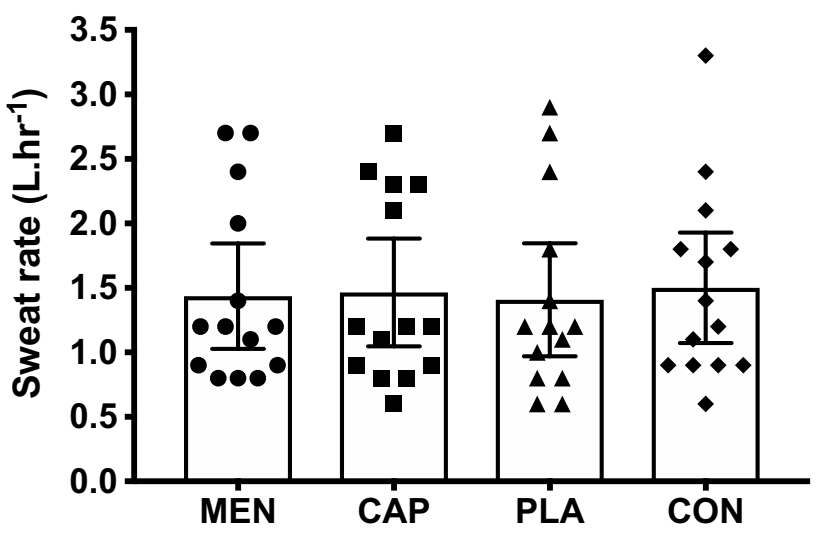

Fig. 5 Mean $\pm 95 \%$ CI sweat rate across the menthol, capsaicin, carbohydrate, and water mouth-rinse conditions

(Sönmez et al. 2010), $20 \mathrm{~km}$ (Riera et al. 2014), and $30 \mathrm{~km}$ cycle TT (Riera et al. 2016). It is noteworthy that improved thermal perception (Riera et al. 2014) and perceived exertion (Riera et al. 2016) do not necessarily improve performance. In support of these equivocal data relating to the importance of thermal/exercise perception on performance following oral menthol, it has also been observed that menthol spray to the torso offers no ergogenic effect during $16.1 \mathrm{~km}$ (Barwood et al. 2015) or $40 \mathrm{~km}$ cycle TT performance (Barwood et al. 2012), or during a pre-loaded $5 \mathrm{~km}$ running TT (Barwood et al. 2014) despite improved thermal sensation. Combining menthol with neck cooling also elicits no difference in exercise performance (time to task failure) in comparison to abdominal or non-menthol neck cooling, or in comparison a control trial, in spite of improved thermal sensation [vs no intervention (Bright et al. 2018)]. The role of improved thermal or exertional perception via menthol remains equivocal with no consensus on which perceptual metric for thermal/exertion feelings is most affected by L-menthol despite their proposed importance during continuous, fixedintensity tasks (Flood 2018). Our data highlight that, during intermittent sprint exercise, in spite of improved thermal perception of equivalent magnitudes to others [specifically thermal comfort, but not thermal sensation as per (Flood et al. 2017)], menthol elicits no ergogenic benefit.

Only one experiment has considered the role of L-menthol and subsequent manipulations in thermal perception on intermittent exercise performance (Trong et al. 2015). The intermittent exercise in this study was unaffected by L-menthol; however, the long, higher intensity intermittent bouts in this study do not reflect the activity profiles of field-based team sports making comparisons with the current protocol inappropriate. A number of other experiments have implemented supramaximal intermittent sprint exercise (similar to the CISP) and altered either thermal perception, i.e., sensation or comfort, or physiological temperature. During intermittent sprint exercise in the heat for example, ice slurry consumption reduced core temperature (start of protocol $-0.5{ }^{\circ} \mathrm{C}$, end of protocol $-0.3{ }^{\circ} \mathrm{C}$ ) and thermal sensation $(-3)$, but did not influence the total distance covered or speed during jog, run, and sprint phases of the protocol (Gerrett et al. 2017). As skin temperature and RPE were not different, the authors concluded, in congruence with the previous work (Duffield and Marino 2007), that combined alterations in core and skin temperature are necessary for influencing intermittent sprint performance in the heat (Gerrett et al. 2017). The CISP has been utilized in experiments where various manipulations of temperature perception and actual temperature have occurred. It has been observed that, in comparison to control conditions, a cooling intervention using ice packs (local cooling of exercising muscle) can reduce muscle temperature (ice packs $-0.6^{\circ} \mathrm{C}$ vs control), core temperature $\left(-0.2^{\circ} \mathrm{C}\right)$, and heart

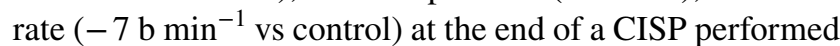
in hot conditions (Castle et al. 2006). The result of these temperature manipulations was increased PP $(+4 \%)$ and WD $(+2 \%)$ in the ice packs only condition (Castle et al. 2006). These performance data, coupled with no differences in either RPE or $T_{\text {sens }}$ between cooling and control trials, suggest that the performance ability during intermittent sprints in the heat is more closely related to physiological responses, and particularly local muscle temperature, rather than perceived temperature. This provides an explanation for why, despite improved thermal comfort with L-menthol, intermittent sprint performance was unchanged in the present study. Support for this mechanism can be found when examining the independent impact of heat acclimation, and pre-cooling interventions on CISP in the heat. Only when implementing a heat acclimation intervention that induces reductions in core temperature $\left(-0.4{ }^{\circ} \mathrm{C}\right)$, heart rate $(-18$ $\mathrm{b} \min ^{-1}$ ) and thermal sensation (-1.0) did PP improve in the heat $(+2 \%)$ with pre-cooling (thermal and non-thermal cooling) offering no additional benefit (Castle et al. 2011). This appears a different mechanism to that observed during $5000 \mathrm{~m}$ running in the heat where pre-cooling demonstrates an ergogenic effect independently (TT duration $-3.7 \%$ ), and when used in conjunction with heat acclimation (TT duration $-7.0 \%$ ) greater performance enhancement than heat acclimation alone (TT duration -6.6\%) (James et al. 2018). These findings highlight that, whilst physiological intervention is a priority for intermittent sprinting in the heat (Castle et al. 2006, 2011), physiological and perceptual manipulations should be conferred for continuous intensity, endurance performance in the heat (James et al. 2017), suggesting that there may still be benefits associated with oral menthol in this domain (Stevens and Best 2017; Flood 2018). 


\section{Experimental considerations and future directions}

In light of the small adjustments to thermal comfort, and no observed change in thermal sensation, it could be suggested that despite replicating dosages utilized in the previous experimental work (Mündel and Jones 2010; Stevens et al. 2016), the concentration of L-menthol $(0.01 \%)$ was insufficient to elicit changes in behavioral thermoregulation. This comment can also be made in regard to the exploratory inclusion of our capsaicin rinse. Recent experimental work has subsequently identified that the concentration of L-menthol (concentrations range 0.05-0.105\% L-menthol at $0.05 \%$ increments when dissolved in ethanol, rather than water) being mouth rinsed does not alter thermal perception within individuals (Best et al. 2018) . Accordingly, although the rinse frequency in the present study replicates the timing used by Mündel and Jones (2010), a more frequent rinse in line with Stevens et al. (2016) may be ergogenic during intermittent sprint and warrants further investigation. Understanding the impact of alterations in L-menthol mouth-rinse temperature may be required to understand the role this plays in intermittent sprint performance given that our experiment utilized a drink temperature similar to deep body temperature to minimize the influence of visceral temperature modulation (Morris et al. 2018), but (for experimental control) this temperature was greater than that likely consumed in actual competition and this may be one reason for different findings compared to studies using cooler mouth rinses (Stevens and Best 2017). It is likely that the capsaicin 'dose' used in this experiment was suboptimal to achieve our experimental aim. The use of capsaicin mouth rinse (to make mechanistic inference rather than as an ergogenic aid) to increase thermal perception, therefore, requires further refinement allied to application, concentration, and frequency given the lack of a response across dependent variables, an opposing finding to that of topical capsaicin (Schlader et al. 2011). The present experiment did not utilize sufficient techniques to understand the mechanisms, and level at which L-menthol cooling is ergogenic. To elucidate this, techniques such as peripheral nerve stimulation and transcranial magnetic stimulation (Goodall et al. 2014; Twomey et al. 2017) may be used to determine central and peripheral components to the reduction in workload associated with intermittent sprint exercise in the heat, with and without alterations in perception via L-menthol. The intensity of continuous endurance activity is closer to the submaximal intensity proposed as subject to influence via pre-cooling (Duffield and Marino 2007), than the supramaximal sprint efforts within the CISP suggesting that perceived temperature plays a lesser role in modulated short sprint exercise of a fixed number and frequency. Performance during submaximal phases of an intermittent sprint protocol is most likely to be influenced by cooling, rather than the sprint itself (Duffield and Marino 2007), Regrettably, this was not quantifiable in the present experiment given that the fixed-intensity nature of our active recovery protocol but the notion that peak sprint performance is less likely to be influenced by actual/perceptual cooling was observed (Fig. 1). These comments raise an important point relating to the protocol used in the present study. Whilst the CISP is a reliable and valid protocol to determine the physiological responses to intermittent sprinting (Hayes et al. 2012, 2014), the specific task structure is such that the CISP is closed in nature. This closed task potentially creates an experimental artifact, whereby participants are not able to sprint freely (in frequency or duration). Future work should consider the benefits of an 'open-ended' task, e.g., devising a protocol with an undefined sprint duration, or participant regulated sprint frequency, performed on a non-motorized treadmill where participants could pace independently in response to nonthermal cooling interventions in the heat. This approach is such that it may facilitate different responses due to the elevation in pacing associated with these tasks, differentiating it from the CISP which implements fixed duration and fixed frequency of sprinting. In light of alterations in both lowand high-intensity running with heat stress (Konefal et al. 2014; Nassis et al. 2015; Watanabe et al. 2017), changes in these movement velocities could also be quantified using this approach in a similar manner to other work (Gerrett et al. 2017). Finally, the CISP is only $40 \mathrm{~min}$ in duration and, therefore, only replicates the first half of a field-based team sport. Future work should, therefore, extend the task duration to more closely replicate the team sport of interest (Turner et al. 2014), in elite team sport players.

\section{Practical implications}

These data suggest that the ergogenic potential of L-menthol associated with endurance tasks does not extend to intermittent sprint performance (of $40 \mathrm{~min}$ ) in very hottemperature conditions $\left(\sim 40{ }^{\circ} \mathrm{C}\right)$. This is in spite of the magnitude in alteration in thermal perception in the present experiment being congruous with others (Stevens et al. 2017; Flood et al. 2017), meaning that our null finding relating to performance was not simply a result of a null finding relating to altering thermal perception as is the case with the capsaicin data. As such, rather than seeking perceptual manipulations alone, individuals seeking to use an intervention to enhance intermittent sprint performance, e.g., team sport players, should preference either pre-cooling, or mid (per) cooling interventions as an acute ergogenic aid (Castle et al. 2006; Luomala et al. 2012; Sunderland et al. 2015), or a heat acclimation strategy, e.g., the preferable isothermic approach (Racinais et al. 2015a; Pryor et al. 2018), to induce enhanced physiological responses to 
intermittent sprinting as part of a chronic intervention (Sunderland et al. 2008; Castle et al. 2011) in a manner that minimizes training disruption (Gibson et al. 2015).

\section{Conclusion}

Mouth rinsing with L-menthol improves thermal comfort during $40 \mathrm{~min}$ of intermittent sprint exercise in the heat, but this does not appear to alter ISP. Capsaicin did not alter thermal perception or ISP. No mouth rinse utilized changed the physiological responses to ISP in the heat. Alterations to the intervention, e.g., mouth-rinse frequency/duration subsequently leading to a greater change in thermal perception, or an open loop ISP may induce ergogenic responses, however, based on the present data; the reduction in ISP over time in hot conditions is not influenced by altering thermal perception.

Acknowledgements We would like to express our gratitude to the participants who voluntarily took part in the experimental study and to Tom Howes, Craig Stout, Atharva Tere, Dominic Mitchell, and Michael Polycarpou for their support with the data collection.

Author contributions ORG and JW conceived and designed research. ORG and MH conducted experiments. JGW analyzed data. ORG, JGW, and MH wrote the manuscript. All authors read and approved the manuscript.

Open Access This article is distributed under the terms of the Creative Commons Attribution 4.0 International License (http://creat ivecommons.org/licenses/by/4.0/), which permits unrestricted use, distribution, and reproduction in any medium, provided you give appropriate credit to the original author(s) and the source, provide a link to the Creative Commons license, and indicate if changes were made.

\section{References}

Barwood MJ, Corbett J, White D, James J (2012) Early change in thermal perception is not a driver of anticipatory exercise pacing in the heat. Br J Sports Med 46:936-942. https://doi.org/10.1136/ bjsports-2011-090536

Barwood MJ, Corbett J, White DK (2014) Spraying with 0.20\% L-menthol does not enhance $5 \mathrm{~km}$ running performance in the heat in untrained runners. J Sports Med Phys Fit 54:595-604

Barwood MJ, Corbett J, Thomas K, Twentyman P (2015) Relieving thermal discomfort: effects of sprayed L-menthol on perception, performance, and time trial cycling in the heat. Scand J Med Sci Sports 25:211-218. https://doi.org/10.1111/sms.12395

Best R, Spears I, Hurst P, Berger N (2018) The development of a menthol solution for use during sport and exercise. Beverages 4:44. https://doi.org/10.3390/beverages4020044

Borg GA (1982) Psychophysical bases of perceived exertion. Med Sci Sports Exerc 14:377-381
Bright FM, Chaseling GK, Jay O, Morris NB (2018) Self-paced exercise performance in the heat with neck cooling, menthol application, and abdominal cooling. J Sci Med Sport. https://doi. org/10.1016/J.JSAMS.2018.09.225

Candas V, Libert JP, Vogt JJ (1979) Human skin wettedness and evaporative efficiency of sweating. J Appl Physiol 46:522-528

Carter JM, Jeukendrup AE, Jones DA (2004) The effect of carbohydrate mouth rinse on 1-h cycle time trial performance. Med Sci Sports Exerc 36:2107-2111

Castle PC, Macdonald AL, Philp A et al (2006) Precooling leg muscle improves intermittent sprint exercise performance in hot, humid conditions. J Appl Physiol 100:1377-1384. https://doi. org/10.1152/japplphysiol.00822.2005

Castle P, Mackenzie RW, Maxwell N et al (2011) Heat acclimation improves intermittent sprinting in the heat but additional pre-cooling offers no further ergogenic effect. J Sports Sci 29:1125-1134

Castle PC, Maxwell N, Allchorn A et al (2012) Deception of ambient and body core temperature improves self paced cycling in hot, humid conditions. Eur J Appl Physiol 112:377-385. https://doi. org/10.1007/s00421-011-1988-y

Cohen J (1992) A power primer. Psychol Bull 112:155-159

Corbett J, Vance S, Lomax M, Barwood MJ (2009) Measurement frequency influences the rating of perceived exertion during submaximal treadmill running. Eur J Appl Physiol 106:311-313. https://doi.org/10.1007/s00421-009-1041-6

Drust B, Waterhouse J, Atkinson G et al (2005) Circadian rhythms in sports performance-an update. Chronobiol Int 22:21-44

Du Bois D, Du Bois EF (1916) A formula to estimate the approximate surface area if height and weight be known. Arch Intern Med $17: 863-871$

Duffield R, Marino FE (2007) Effects of pre-cooling procedures on intermittent-sprint exercise performance in warm conditions. Eur J Appl Physiol 100:727-735. https://doi.org/10.1007/s0042 1-007-0468-x

Duffield R, Green R, Castle P, Maxwell N (2010) Precooling can prevent the reduction of self-paced exercise intensity in the heat. Med Sci Sport Exerc 42:577-584

Durnin JV, Womersley J (1974) Body fat assessed from total body density and its estimation from skinfold thickness: measurements on 481 men and women aged from 16 to 72 years. Br J Nutr 32:77-97

Flood TR (2018) Menthol use for performance in hot environments. Curr Sports Med Rep 17:135-139. https://doi.org/10.1249/ JSR.0000000000000474

Flood TR, Waldron M, Jeffries O (2017) Oral 1-menthol reduces thermal sensation, increases work-rate and extends time to exhaustion, in the heat at a fixed rating of perceived exertion. Eur J Appl Physiol 117:1501-1512. https://doi.org/10.1007/s00421-017-3645-6

Galloway SDR, Maughan RJ (1997) Effects of ambient temperature on the capacity to perform prolonged cycle exercise in man. Med Sci Sport Exerc 29:1240-1249. https://doi.org/10.1097/00005 768-199709000-00018

Garth AK, Burke LM (2013) What do athletes drink during competitive sporting activities? Sport Med 43:539-564. https://doi. org/10.1007/s40279-013-0028-y

Gerrett N, Jackson S, Yates J, Thomas G (2017) Ice slurry ingestion does not enhance self-paced intermittent exercise in the heat. Scand J Med Sci Sports 27:1202-1212. https://doi.org/10.1111/ sms. 12744

Gibson OR, Dennis A, Parfitt T et al (2014) Extracellular Hsp72 concentration relates to a minimum endogenous criteria during acute exercise-heat exposure. Cell Stress Chaperones 19:389-400. https ://doi.org/10.1007/s12192-013-0468-1

Gibson OR, Mee JA, Tuttle JA et al (2015) Isothermic and fixed intensity heat acclimation methods induce similar heat adaptation following short and long-term timescales. J Therm Biol 49-50:55-65 
Gill N, Sleivert G (2001) Effect of daily versus intermittent exposure on heat acclimation. Aviat Space Environ Med 72:385-390

Girard O, Brocherie F, Bishop DJ (2015) Sprint performance under heat stress: a review. Scand J Med Sci Sports 25:79-89. https:// doi.org/10.1111/sms. 12437

Girard O, Billaut F, Christian RJ et al (2017) Exercise-related sensations contribute to decrease power during repeated cycle sprints with limited influence on neural drive. Eur J Appl Physiol 117:2171-2179. https://doi.org/10.1007/s00421-017-3705-y

González-Alonso J (2012) Human thermoregulation and the cardiovascular system. Exp Physiol 97:340-346. https://doi.org/10.1113/ expphysiol.2011.058701

González-Alonso J, Crandall CG, Johnson JM (2008) The cardiovascular challenge of exercising in the heat. J Physiol 586:45-53. https ://doi.org/10.1113/jphysiol.2007.142158

Goodall S, Howatson G, Romer L, Ross E (2014) Transcranial magnetic stimulation in sport science: a commentary. Eur J Sport Sci 14(Suppl 1):S332-S340. https://doi.org/10.1080/17461 391.2012.704079

Guy JH, Deakin GB, Edwards AM et al (2014) Adaptation to hot environmental conditions: an exploration of the performance basis, procedures and future directions to optimise opportunities for elite athletes. Sports Med 45:303-311. https://doi.org/10.1007/s4027 9-014-0277-4

Hayes M, Smith D, Castle PC et al (2012) Peak power output provides the most reliable measure of performance in prolonged intermittent-sprint cycling. J Sports Sci 37-41. https://doi. org/10.1080/02640414.2012.744077

Hayes M, Castle PC, Ross EZ, Maxwell NS (2014) The influence of hot humid and hot dry environments on intermittent-sprint exercise performance. Int J Sports Physiol Perform 9:387-396. https://doi. org/10.1123/ijspp.2012-0247

James CA, Hayes M, Willmott AGB et al (2017) Defining the determinants of endurance running performance in the heat. Temperature 4:314-329. https://doi.org/10.1080/23328940.2017.1333189

James CA, Richardson AJ, Willmott AG et al (2018) Short-term heat acclimation and precooling, independently and combined, improve 5 $\mathrm{km}$ running performance in the heat. J Strength Cond Res 32:13661375. https://doi.org/10.1519/JSC.0000000000001979

Jeffries O, Goldsmith M, Waldron M (2018) L-menthol mouth rinse or ice slurry ingestion during the latter stages of exercise in the heat provide a novel stimulus to enhance performance despite elevation in mean body temperature. Eur J Appl Physiol. https://doi.org/10.1007/ s00421-018-3970-4

Kenefick RW, Cheuvront SN, Sawka MN (2007) Thermoregulatory function during the marathon. Sports Med 37:312-315

Konefal M, Chmura P, Andrzejewski M, Chmura J (2014) Analysis of motor performance of professional soccer players in different environmental conditions. Trends Sport Sci 4:221-227

Lee JKW, Shirreffs SM (2007) The influence of drink temperature on thermoregulatory responses during prolonged exercise in a moderate environment. J Sports Sci 25:975-985. https://doi. org/10.1080/02640410600959947

Lei T-H, Stannard SR, Perry BG et al (2016) Influence of menstrual phase and arid vs. humid heat stress on autonomic and behavioural thermoregulation during exercise in trained but unacclimated women. J Physiol. https://doi.org/10.1113/JP273176

Luomala MJ, Oksa J, Salmi JA et al (2012) Adding a cooling vest during cycling improves performance in warm and humid conditions. J Therm Biol 37:47-55. https://doi.org/10.1016/J.JTHER BIO.2011.10.009

Maxwell NS, Aitchison TC, Nimmo MA (1996) The effect of climatic heat stress on intermittent supramaximal running performance in humans. Exp Physiol 81:833-845

Mee JA, Gibson OR, Doust JJH, Maxwell NS (2015) A comparison of males and females' temporal patterning to short- and long-term heat acclimation. Scand J Med Sci Sports 25:250-258. https://doi. org/10.1111/sms.12417

Mohr M, Nybo L, Grantham J, Racinais S (2012) Physiological responses and physical performance during football in the heat. PLoS One 7:e39202. https://doi.org/10.1371/journal.pone.0039202

Montell C, Caterina MJ (2007) Thermoregulation: channels that are cool to the core. Curr Biol 17:R885-R887. https://doi.org/10.1016/j. cub.2007.08.016

Morris NB, Chaseling GK, Bain AR, Jay O (2018) The temperature of water ingested before exercise alters the onset of physiological heat loss responses. Am J Physiol Integr Comp Physiol. https://doi. org/10.1152/ajpregu.00028.2018

Mündel T, Jones DA (2010) The effects of swilling an 1(-)-menthol solution during exercise in the heat. Eur J Appl Physiol 109:59-65. https ://doi.org/10.1007/s00421-009-1180-9

Nassis GP, Brito J, Dvorak J et al (2015) The association of environmental heat stress with performance: analysis of the 2014 FIFA World Cup Brazil. Br J Sports Med 49:609-613. https://doi.org/10.1136/bjspo rts-2014-094449

Nybo L, Girard O, Mohr M et al (2013) Markers of muscle damage and performance recovery after exercise in the heat. Med Sci Sports Exerc 45:860-868. https://doi.org/10.1249/MSS.0b013e31827ded0 4

Pryor JL, Johnson EC, Roberts WO, Pryor RR (2018) Application of evidence-based recommendations for heat acclimation: individual and team sport perspectives. Temperature. https://doi.org/10.1080/23328 940.2018.1516537

Racinais S, Alonso JM, Coutts AJ et al (2015a) Consensus recommendations on training and competing in the heat. Scand J Med Sci Sports 25:6-19. https://doi.org/10.1111/sms.12467

Racinais S, Périard JD, Karlsen A, Nybo L (2015b) Effect of heat and heat-acclimatization on cycling time-trial performance and pacing. Med Sci Sports Exerc 47:601-606. https://doi.org/10.1249/ MSS.0000000000000428

Ramanathan NL (1964) A new weighting system for mean surface temperature of the human body. J Appl Physiol 19:531-533

Riera F, Trong TT, Sinnapah S, Hue O (2014) Physical and perceptual cooling with beverages to increase cycle performance in a tropical climate. PLoS One 9:e103718. https://doi.org/10.1371/journ al.pone. 0103718

Riera F, Trong T, Rinaldi K, Hue O (2016) Precooling does not enhance the effect on performance of midcooling with ice-slush/menthol. Int J Sports Med 37:1025-1031. https://doi.org/10.1055/s-0042-107597

Sawka MN, Burke LM, Eichner ER et al (2007) American College of Sports Medicine position stand. Exercise and fluid replacement. Med Sci Sports Exerc 39:377-390. https://doi.org/10.1249/ mss.0b013e31802ca597

Schlader ZJ, Simmons SE, Stannard SR, Mündel T (2011) The independent roles of temperature and thermal perception in the control of human thermoregulatory behavior. Physiol Behav 103:217-224. https://doi.org/10.1016/j.physbeh.2011.02.002

Siri WE (1956) The gross composition of the body. Adv Biol Med Phys 4:239-280

Sönmez G, Colak M, Sönmez S, Schoenfeld B (2010) Effects of oral supplementation of mint extract on muscle pain and blood lactate. Biomed Hum Kinet 2:66-69. https://doi.org/10.2478/v1010 $1-0016-8$

Stevens CJ, Best R (2017) Menthol: a fresh ergogenic aid for athletic performance. Sport Med 47:1035-1042. https://doi.org/10.1007/ s40279-016-0652-4

Stevens CJ, Thoseby B, Sculley DV et al (2016) Running performance and thermal sensation in the heat are improved with menthol mouth rinse but not ice slurry ingestion. Scand J Med Sci Sports. https:// doi.org/10.1111/sms.12555

Stevens CJ, Bennett KJM, Sculley DV et al (2017) A comparison of mixed-method cooling interventions on preloaded running 
performance in the heat. J Strength Cond Res 31:620-629. https:// doi.org/10.1519/JSC.0000000000001532

Sunderland C, Morris JG, Nevill ME (2008) A heat acclimation protocol for team sports. Br J Sports Med 42:327-333. https://doi. org/10.1136/bjsm.2007.034207

Sunderland C, Stevens R, Everson B, Tyler CJ (2015) Neck-cooling improves repeated sprint performance in the heat. Front Physiol. https://doi.org/10.3389/fphys.2015.00314

Toner MM, Drolet LL, Pandolf KB (1986) Perceptual and physiological responses during exercise in cool and cold water. Percept Mot Skills 62:211-220

Trong TT, Riera F, Rinaldi K et al (2015) Ingestion of a cold temperature/menthol beverage increases outdoor exercise performance in a hot, humid environment. PLoS One 10:e123815. https://doi. org/10.1371/journal.pone.0123815
Turner G, Gibson OR, Maxwell NS (2014) Simulated moderate hypoxia reduces intermittent sprint performance in games players. J Sports Med Phys Fit 54:566-574

Twomey R, Wrightson JG, Fletcher H et al (2017) Exercise-induced fatigue in severe hypoxia after an intermittent hypoxic protocol. Med Sci Sport Exerc 49:2422-2432. https://doi.org/10.1249/MSS.00000 00000001371

Watanabe N, Wicker P, Yan G (2017) Weather conditions, travel distance, rest, and running performance: the 2014 FIFA World Cup and implications for the future. J Sport Manag 31:27-43. https:// doi.org/10.1123/jsm.2016-0077

Publisher's Note Springer Nature remains neutral with regard to jurisdictional claims in published maps and institutional affiliations. 